\title{
Genomic analysis of circular RNAs in heart
}

\author{
Kunzhe Dong ${ }^{1}$, Xiangqin $\mathrm{He}^{1}$, Huabo Su ${ }^{1,2}$, David J. R. Fulton ${ }^{1,2}$ and Jiliang Zhou ${ }^{1 *}$ (D)
}

\begin{abstract}
Background: Heart failure is a leading cause of human morbidity and mortality. Circular RNAs (circRNAs) are a newly discovered class of RNA that have been found to have important physiological and pathological roles. In the current study, we de novo analyzed existing whole transcriptome data from 5 normal and 5 dilated cardiomyopathy (DCM) human heart samples and compared the results with circRNAs that have been previously reported in human, mouse and rat hearts.

Results: Our analysis identifies a list of cardiac circRNAs that are reliably detected in multiple studies. We have also defined the top 30 most abundant circRNAs in healthy human hearts which include some with previously unrecognized cardiac roles such as circHIPK3_11 and circTULP4_1. We further found that many circRNAs are dysregulated in DCM, particularly transcripts originating from DCM-related gene loci, such as TTN and RYR2. In addition, we predict the potential of cardiac circRNAs to sponge miRNAs that have reported roles in heart disease. We found that cirCALMS1_6 has the highest potential to bind miR-133, a microRNA that can regulate cardiac remodeling. Interestingly, we detected a novel class of circRNAs, referred to as read-though ( $\mathrm{rt}$ )-circRNAs which are produced from exons of two different neighboring genes. Specifically, rt-circRNAs from SCAF8 and TIAM2 were observed to be dysregulated in DCM and these rt-circRNAs have the potential to sponge multiple heart disease-related miRNAs.
\end{abstract}

Conclusions: In summary, this study provides a valuable resource for exploring the function of circRNAs in human heart disease and establishes a functional paradigm for identifying novel circRNAs in other tissues.

Keywords: Circular RNA, Dilated cardiomyopathy, Read-through circRNA, miRNA sponge

\section{Background}

Heart failure (HF) is a leading cause of human morbidity and mortality worldwide. Despite advances in the management of HF, it remains a tremendous and growing public health burden in an aging population with an estimated prevalence of $>37.7$ million individuals globally [1]. Dilated cardiomyopathy (DCM), characterized by the dilation and impaired contraction of the left or both ventricles, is a primary cause of HF and sudden cardiac death [2]. Understanding the etiology of DCM is vitally

\footnotetext{
*Correspondence: jizhou@augusta.edu

${ }^{1}$ Department of Pharmacology and Toxicology, Medical College

of Georgia, Augusta University, 1459 Laney Walker Blvd, Augusta, GA

30912, USA

Full list of author information is available at the end of the article
}

important in the search for better therapeutic approaches for HF.

In addition to non-genetic causes, such as hypertension, inflammation, and toxins, genetic factors are increasingly recognized for their roles in HF susceptibility $[3,4]$. Currently, mutations in more than 100 genes have been linked to DCM [5]. However, these DCM relevant gene variants are only detected in $~ 37 \%$ of DCM patients [6]. Therefore, it is likely that other unknown genetic factors await discovery. The aberrant expression of different types of RNA transcripts, including those transcribed from protein coding genes [7-9], long noncoding genes $[10,11]$ and miRNAs [12-14], have been observed in DCM.

Circular RNAs (circRNAs) are a novel class of RNA that are generated by the covalent ligation of the $5^{\prime}$ and $3^{\prime}$ ends of a single-stranded RNA transcript to form a 
circular transcript [15]. CircRNAs are stable and resistant to RNase R, widespread throughout the plant and animal kingdoms and conserved across multiple species. Furthermore, circRNAs can be expressed in a tissue-specific manner [16, 17]. These properties make circRNAs excellent candidates as potential biomarkers for the diagnosis of disease and therapeutic intervention. Emerging evidence has demonstrated that circRNAs play important roles in various physiological and pathological processes, such as myogenesis [18], phenotypic modulation of smooth muscle cells $[19,20]$, atherosclerosis [21, 22], and cancer [23-25].

Although several efforts have been made to characterize the circRNAs expressed in the heart [26-29], our understanding of the importance of cardiac circRNAs is still very limited. Furthermore, several fundamental questions remain to be addressed. First, there is a large discrepancy between the presence and abundance of cardiac circRNAs that have been identified in previous studies [27-29], which limits any interpretation of their importance. To pinpoint truly important cardiac circRNAs, a cross-reference analysis between multiple independent studies is required. Second, it has been documented that circRNAs function primarily as molecular sponges to competitively bind miRNAs or encode small peptides. While the coding ability of cardiac circRNAs have been investigated recently by analyzing translational Ribo-seq data from human hearts [28], the potential of cardiac circRNAs to function as miRNA sponges remains incompletely understood. Finally, read through-circRNAs (rt-circRNAs), a recently discovered type of circRNAs that are produced from the exons of two adjacent genes on the same strand, have been implicated in both normal and tumor tissues [30], yet whether this type of rt-circRNAs are expressed in the human heart remains to be identified.

To address these questions, we de novo analyzed publicly available RNA-seq datasets from human hearts. These data sets were of sufficient sample size (5 normal controls and 5 DCM patients) [10], to enable us to investigate the general landscape of human cardiac circRNAs under homeostatic (normal) and disease settings. By integrating the analysis of circRNAs identified in human hearts with that of mouse and rat hearts in previous studies [26, 27, 29], we: (i) characterized the general features of circRNAs in human hearts and compared the identified circRNAs with circRNAs reported by previous studies and databases; (ii) determined the key circRNAs expressed in healthy hearts as defined by the top 30 most abundant circRNAs; (iii) identified circRNAs that are dysregulated in DCM hearts; (iv) predicted the potential of high-confidence cardiac circRNAs to act as a sponge for previously identified heart disease-related miRNAs;
Lastly, we identified a list of rt-circRNAs, especially those from the SCAF8 and TIAM2 gene loci that are dysregulated in DCM and show strong potential of being miRNA sponges. Our work identifies a set of highly reliable circRNAs with potentially important roles in maintaining cardiac homeostasis which is a valuable resource for exploring the function of circRNAs in human heart disease. Our work further establishes a paradigm for identifying novel circRNAs in other tissues.

\section{Methods \\ Identification of circRNAs}

RNA-seq raw reads from the left ventricle of 5 DCM patients and 5 normal controls were obtained from SRA database (SRP108571) [10] and analyzed as illustrated in Additional file 1: Figure S1. Low-quality reads were first removed by Trimmomatic 3.0 [31]. The clean reads were then mapped to the GRCh38 human genome reference by Bowtie2 [32]. For the linear RNA analysis, the raw count of each gene was calculated by using HTSeq [33] and Fragments Per Kilobase of exon per Million fragments mapped (FPKM) values were calculated from raw counts to quantify expression of genes [34]. Differential analysis of linear RNA between DCM and normal samples was performed with DESeq2 [35] and genes with fold change $(\mathrm{FC})>2$ and $\mathrm{FDR}<0.05$ were considered significance. Next, circRNAs were identified following the instruction of find_circ [36]. Briefly, by using SAMtools the unmapped reads were retrieved [37]. Then these unmapped reads were cut off 20 mers from both ends and mapped to the human genome independently in order to find unique anchor positions. Anchors that aligned in the reversed orientation suggest a back-spliced junction. CircRNAs with $\geq 2$ unique back spliced reads in at least one sample were saved for analysis of circRNA features. The expression of circRNAs was normalized to Spliced Reads per Billion Mapped Reads (SRPBM). For identification of the differentially expressed circRNAs between normal and DCM samples, only those high confidence circRNAs, with $\geq 2$ unique reads in at least four samples from at least one group were used. Differentially expressed circRNAs were identified with edgeR package based on a negative binomial distribution model [38]. CircRNAs with $\mathrm{FC}>2$ and $p$ value $<0.05$ were considered significant. Pathway analysis was performed using Metascape [39].

\section{Comparison with other studies}

Results from three previous studies [26-29], which identified circRNAs in human, mouse or rat hearts, as well as four public circRNA databases, including circBase [40], circBank [41], circRNADB [42], CIRCpedia V2 [43], were used in comparison with our results. 
The R package liftOver (https://www.bioconductor.org/ packages/release/workflows/html/liftOver.html) was used to convert start and end coordinates (hg19, mm9, mm10 and rn5) to hg38 first and then the converted coordinates were used for cross-reference and crossspecies comparison. The venn diagrams were generated with online tool (https://bioinformatics.psb.ugent.be/ webtools/Venn/).

\section{Analysis of circRNAs from two different genes}

The 50-bp sequences from each side of the back-spliced junction of the cricRNAs arising from the exons of two different genes (gene 1 and gene 2), were retrieved and used as query sequences to perform BLAST analysis against human cDNA sequences downloaded from ENSEMBL database with default parameters. If any of the two sequences could be aligned to both gene 1 and gene 2 , the circRNA was considered as possible false positive due to the sequence homologue of gene 1 and gene 2 . If the sequence from each side of the back-spliced junction returned BLAST hits only matching its expected host gene, the circRNA was considered as true rt-circRNA.

Construction of circRNA-miRNA-mRNA regulatory network A list of 50 miRNAs which have been implicated in heart disease were obtained (References were provided in Additional file 2: Table S1). Seed sequence and potential targeted genes of these miRNAs was obtained from TargetScan database (https://www.targetscan.org/). CircRNA sequence was retrieved using custom $\mathrm{R}$ script and TargetScan was used for predicting the binding sites of the selected miRNAs. CircRNAs with at least 5 binding sites for at least one miRNA were considered to have potential to sponge miRNAs. The circRNAmiRNA-mRNA regulatory network was visualized using Cytoscape (https://cytoscape.org/).

\section{Experimental validation of circRNAs}

Due to the lack of availability of human samples, the mouse orthologs of 6 identified human circRNAs were used for experimental validation. Mouse heart RNA was extracted with TRIzol reagent (Invitrogen) and reverse transcribed using random hexamers and High Capacity RNA-to-cDNA Kit (Invitrogen). Convergent primers with opposite orientation were designed (Additional file 3: Table S2) and used for PCR amplification on mouse heart cDNA and genomic DNA. Mouse Yap1 gene primers were used as a positive control for PCR amplification of genomic DNA. PCR products were then subjected to Sanger sequencing.

\section{Results}

\section{General features of circRNAs in both healthy and DCM} human hearts

We performed a de novo analysis of public RNA-seq data sets that were generated from left ventricular tissues obtained from 5 healthy individuals and 5 DCM patients [10]. These data sets were selected because they were generated using a ribosome-depletion library, which is the gold standard method to identify back-spliced junctions for circRNAs detection [44]. We first removed lowquality reads and then utilized Bowtie2 [32] to align clean reads against the human reference genome. Reads continually aligned to the genome were used for linear gene expression analysis and the remaining un-mapped reads were further used as input for find_circ [36] to detect both linear and back-spliced junctions (Additional file 1: Figure S1). We first performed a quality control validation of this data by performing principal component analysis (PCA) based on the expression of linear genes with raw count $>10$ in at least 5 samples. PCA analysis confirmed that samples from each group were clustered respectively as expected (Additional file 4: Figure S2A). We further examined the expression of a marker of $\mathrm{HF}$, atrial natriuretic peptide $\alpha(N P P A)$. Consistent with the reported HF phenotype [45], our analysis revealed that the expression of NPPA is significantly increased in DCM specimens (Additional file 4: Figure S2B). Taken together, these initial analyses confirm the quality of this RNAseq dataset and its suitability for the further analysis of circRNAs.

With a threshold cutoff of $\geq 2$ unique back-spliced reads present in at least one sample, we identified a total of 20,214 circRNAs in the 10 samples (Fig. 1a and Additional file 5: Table S3). The identified circRNAs originated primarily from the coding-sequence (CDS) regions of exons of single linear genes (67.2\%), followed by exons spanning coding and untranslated regions (UTR) (UTRCDS) (11.6\%) (Fig. 1b). Notably, a total of 252 circRNAs (1.2\%) were assigned to the exons of two adjacent genes on the same strand (Additional file 6: Table S4), likely representing a novel class of circRNAs termed as rt-circRNA [23]. In particular, the genes $M Y H 6$ and $M Y H 7$ produced the largest number $(\mathrm{n}=22)$ of such circRNAs, which were also observed in human, mouse and rat hearts as reported by two earlier studies (Additional file 7: Figure S3A-C) [27, 29]. In addition, these MYH6/ $M Y H 7$-derived circRNAs were highly abundant and conserved in hearts of human, mouse and rat (Additional file 7: Figure S3D). However, close inspection of the sequence between the exons where they are back-spliced suggests that the back-spliced junctions of these circRNAs are probably artifactual due to read misalignment caused by the high sequence homology between MYH6 


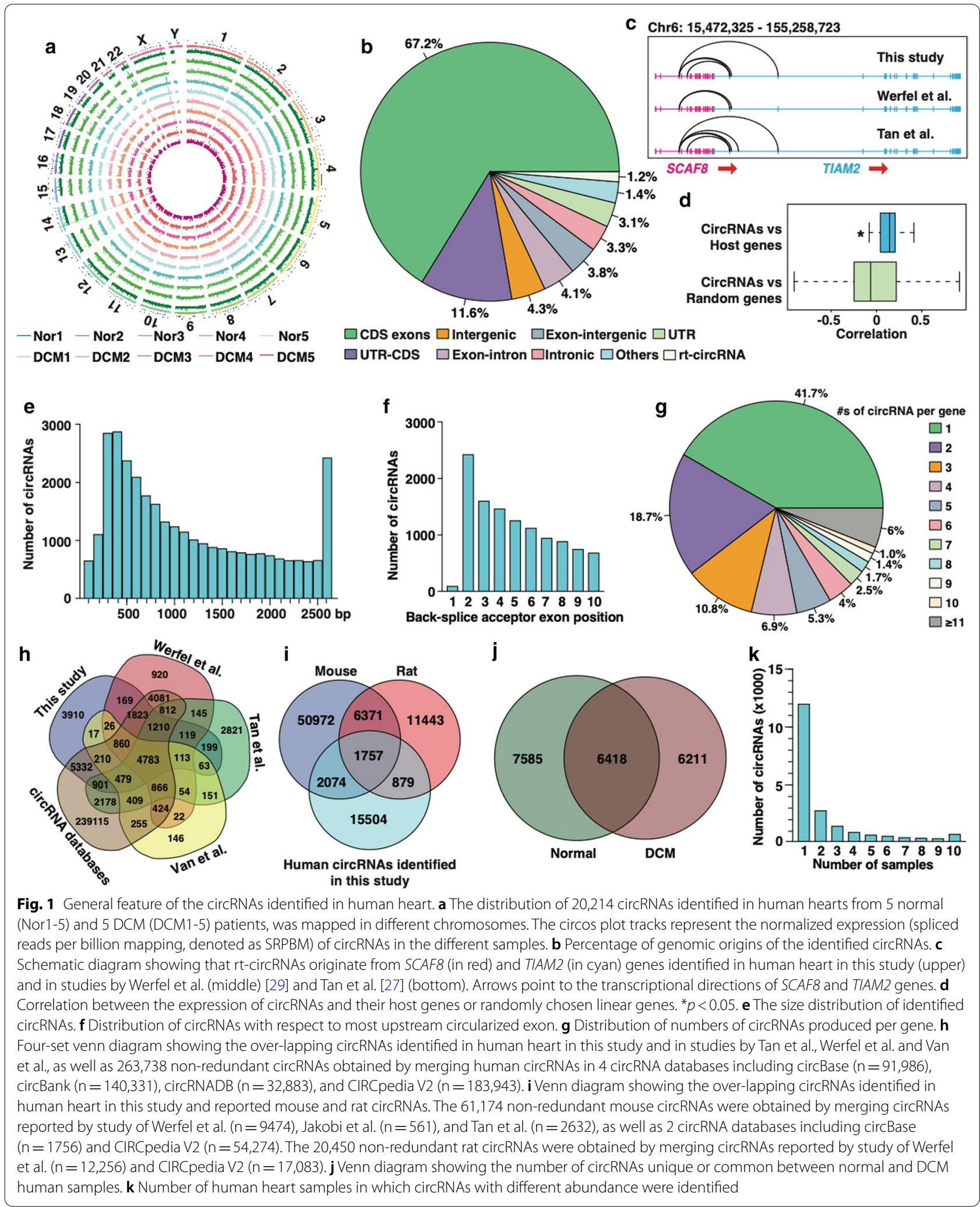


and $M Y H 7$ (Additional file 7: Figure S3E). This result prompted us to employ more stringent criteria to filter circRNAs originating from two neighboring genes with highly homologous sequences. Using this revised filtering strategy, we identified 110 putative rt-circRNAs. Among them, SCAF8 and TIAM2 contain the largest number of rt-circRNAs $(\mathrm{n}=4)$, all of which were also previously identified in the human heart (Fig. 1c).

We next examined whether there was a correlative relationship between the expression of circRNAs and their parent linear genes. We found that there was a significantly higher correlation between circRNAs and their linear parent genes compared to that between circRNAs and randomly chosen linear genes (Fig. 1d), suggesting that the generally high-abundance circRNAs tend to have high-abundance linear counterparts. We further found that the length of the majority of identified circRNAs was around 300-400 bp (Fig. 1e). In accordance with previous studies [46-48], the most common splice accepting circularized exon was exon 2 (Fig. 1f). Furthermore, we found that the majority of circRNA-producing genes generated 1 circRNA $(41.7 \%, 2,231 / 5,350)$ and only $6.0 \%$ $(\mathrm{n}=319)$ produced more than 10 circRNAs (Fig. 1g). The top two genes producing the largest number of circRNAs were TTN (Titin) and RYR2 (Ryanodine receptor 2), which generated 197 and 173 circRNAs, respectively (Additional file 8: Figure S4A-B). Because the TTN and $R Y R 2$ genes have the largest number of exons in human genome, we hypothesize that the number of circRNAs produced is positively correlated to the number of exons within their parent genes. Indeed, a proportional increase in the number of circRNAs was observed to correlate with the number of exons per gene (Additional file 8: Figure S4C). Furthermore, we found 17,733 identified circRNAs, in total, contain exons. Among these, single exonic circRNAs were found in $8.4 \%(1494 / 17,733)$ while the rest of circRNAs were encoded by more than two exons (Additional file 8: Figure S4D).

Next we compared the circRNAs that we identified with others that were previously identified in human hearts [27-29] and enlisted in circRNA databases [40, $41,43,49]$. We found that 16,304 circRNAs $(80.7 \%)$ were previously reported by either human heart circRNA studies or enlisted in circRNA databases (Fig. 1h). In addition, 3,831 and 2,636 circRNAs have orthologs in the mouse and rat, respectively. Importantly, 1,757 were conserved in all three species by integrating mouse and rat circRNAs reported by previous studies and databases $[26,27$, 29, 40, 41, 43, 49] (Fig. 1I). We further found that 7,585 and 6,211 circRNAs were specifically identified in normal and DCM hearts, respectively, and that 6,418 were identified in both (Fig. 1j). Further analysis revealed that only 729 circRNAs were present in all the 10 samples
(Fig. 1k), suggesting that many circRNAs exhibit a high degree of variation in expression among human individuals. Additionally, the majority of circRNAs $(n=12,099)$ were observed in only one sample (Fig. 1k), which likely represent false positives or low-abundance circRNAs. To clarify this, we compared the circRNAs observed once in this study with circRNAs reported by 3 previous human heart circRNA studies [27-29] and 4 circRNA databases $[40,41,43,49]$. This analysis revealed that $8,666(71.6 \%)$ among them were previously detected (Additional file 9: Figure S5), suggesting these circRNAs observed in a single sample are probably authentic and thus should be retained in studies aiming for circRNA discovery. Collectively, these results indicate that the expression of circRNAs, including rt-circRNAs, is widespread in the human heart with a certain degree of conservation between species.

\section{Identification of the most abundant circRNAs in healthy human heart}

Given the observation that circRNA abundance was not universally distributed across samples (Fig. 1k), we restricted our analysis to 2,461 circRNAs which were considered to be high-confidence circRNAs as they had $\geq 2$ reads spanning the back-spliced junction in $\geq 4$ samples in at least one group (Additional file 10: Table S5). All these high-confidence circRNAs were overlapped with previously identified circRNAs (Additional file 11: Figure S6A) and 1,121 (45.6\%) showed conservation in either mouse or rat (Additional file 11: Figure S6B), indicating the reliability of these circRNAs we identified.

CircRNAs that are abundantly expressed in normal heart likely contribute to the maintenance of cardiac homeostasis. We therefore focused on the top 30 circRNAs with the highest average expression level across the 5 healthy heart samples (Fig. 2a). The top 2 most abundant circRNAs (circSLC8A1_11 and circSLC8A1_12) originate from the SLC8A1 (sodium/ calcium exchanger 1) gene. While most of the top circRNAs were also identified in the studies of Tan et al. [27] and Werfel et al. [29], which found high expression levels of circRNAs such as circSLC8A1_12, circHIPK3_1 and circALPK2_2 in healthy hearts, some exceptions were observed. These include circSLC8A1_11 and circYY1AP1_3 that were not detected in study of Tan et al. [27], as well as circZNF91_2 and circPCMTD1_6 whose expression was identified to be abundant in the heart in only one of the previous studies (Fig. 2a). Comparison of the results obtained from different species revealed that half of these highly abundant circRNAs in normal human hearts were conserved in mouse or rat hearts, but only 2 of them including circSLC8A1_11 and circHIPK3_1 exhibited high levels of expression 


\begin{tabular}{|c|c|c|c|c|c|c|}
\hline \multirow{2}{*}{$\begin{array}{l}\text { a } \\
\quad \text { circSLC8A1_12 }\end{array}$} & \multicolumn{3}{|c|}{ Human } & \multicolumn{2}{|c|}{ Mouse } & \multirow[t]{2}{*}{ Rat } \\
\hline & 1 & & & & & \\
\hline circSLC8A1_11 & 2 & & 22 & & 19 & 21 \\
\hline circHIPK3_1 & 3 & & & 29 & 26 & 80 \\
\hline circN4BP2L2_13 & 4 & 23 & 3 & & 1365 & 519 \\
\hline circMB_1 & 5 & 20 & & & & \\
\hline circZNF91_2 & & 5 & 2824 & & & \\
\hline circEXOC6B_13 & & & 4 & 858 & 543 & 4848 \\
\hline circRHOBTB3_13 & & 28 & & & & \\
\hline circALPK2__2 & & & 12 & 706 & & 1464 \\
\hline circTTN_73 & & 15 & 804 & & & \\
\hline circPCMTD1_6 & 11 & 151 & 31 & 487 & 540 & 1825 \\
\hline circBPTF_25 & 12 & 31 & 383 & & & \\
\hline circQKI_4 & 13 & 53 & & 83 & 50 & \\
\hline circTULP4_1 & 14 & & 320 & 17 & 16 & 6472 \\
\hline circQKI_2 & 15 & 67 & 11 & 818 & 531 & \\
\hline circSMARCA5_4 & 16 & 58 & 14 & & & \\
\hline circYY1AP1_3 & 17 & & 57 & & & \\
\hline circSPHKAP_1 & 18 & 118 & 548 & & & \\
\hline circFNDC3B_8 & 19 & 84 & 28 & & & 1436 \\
\hline circMYBPC3_3 & 20 & 25 & - & & & \\
\hline circLIFR_9 & 21 & 70 & 54 & & & 6029 \\
\hline circFCHO2 5 & 22 & 180 & 49 & & & \\
\hline circCOR01C_1 & 23 & 97 & & & & \\
\hline circARHGAP5_2 & 24 & 17 & 7013 & 120 & 8840 & 10881 \\
\hline circLYYPLAL1_6 & 25 & & 37 & & & \\
\hline circRMST_3 & 26 & 406 & 503 & & 1272 & \\
\hline circMTUS1_17 & 27 & 76 & 883 & 167 & 4897 & \\
\hline circANKRD12_5 & 28 & 89 & 327 & 513 & & \\
\hline circSETD3_3 & 29 & 79 & 34 & 286 & & \\
\hline \multirow[t]{2}{*}{ circARHGAP5_1 } & 30 & 26 & 14159 & 250 & & 6243 \\
\hline & 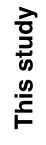 & $\begin{array}{l}\dot{\pi} \\
\frac{\pi}{0} \\
\frac{1}{\pi} \\
\stackrel{-}{-}\end{array}$ & 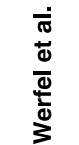 & 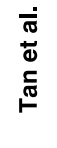 & 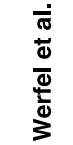 & 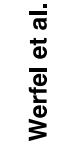 \\
\hline
\end{tabular}
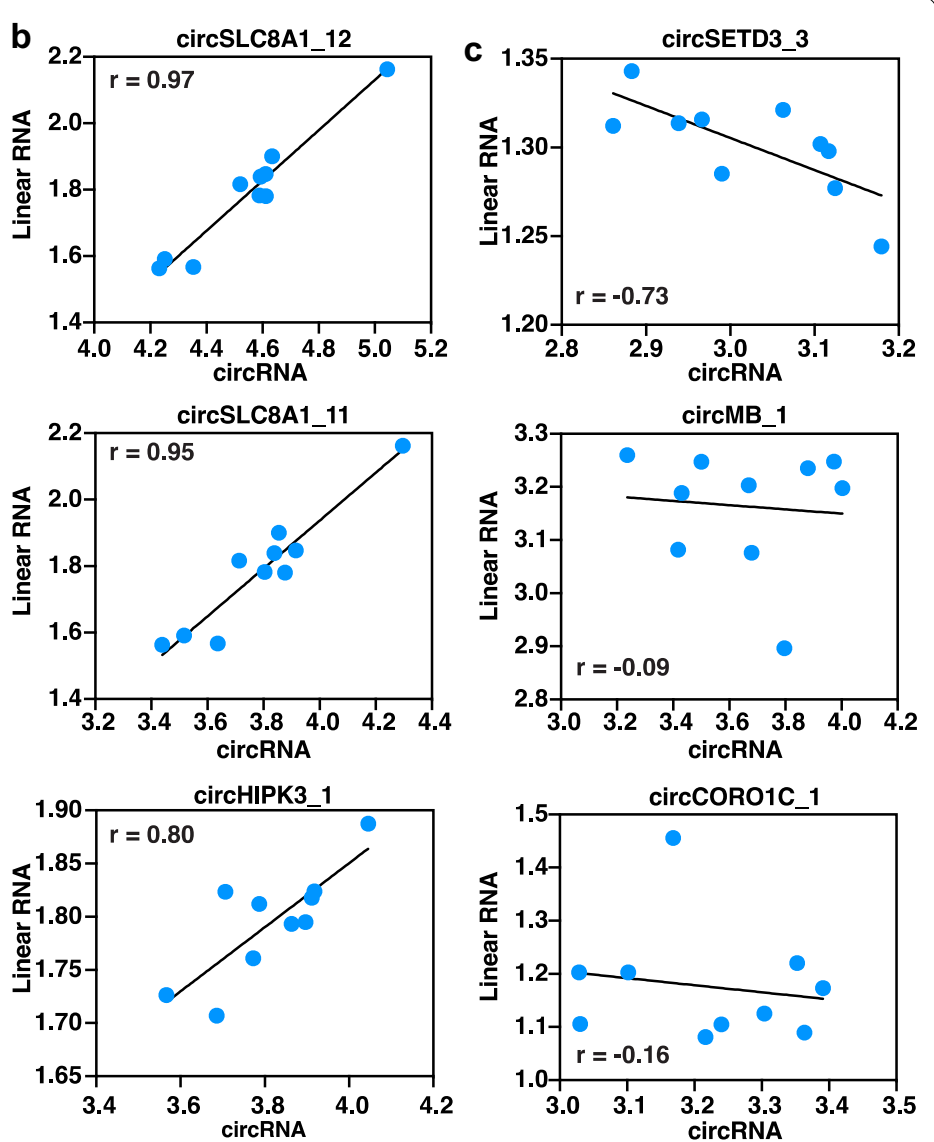

Fig. 2 Identification of the most abundant circRNAs in normal human hearts. a Heatmap illustrates the ranking of the top 30 most abundant circRNAs identified in normal hearts in this study, and in normal human, mouse and rat hearts that were identified by Tan et al. and Werfel et al. [27, 29]. The numbers in the colored boxes indicate the ranking of the circRNAs within the indicated species and blank boxes represent the circRNA was not detected in the indicated species. b Correlation between expression of the top 3 most abundant circRNAs including circSLC8A1_12, circSLC8A1_11 and cirCHIPK3_1 and their host genes which show positively correlation, as well as c circSETD3_3, circMB_1, circCORO1C_1 and their host genes which are independent of each other

in all species, and 2 of them including circQKI_4 and circTULP4_1 were abundantly expressed in human and mouse hearts, but not in rat. The expression levels of the remaining conserved circRNAs were moderate or low in rodent hearts. We then calculated the correlation between the abundance of top circRNAs and their host genes and found a highly positive correlation for most of these circRNAs (Additional file 12: Figure S7A), including the top 3 most abundant ones (i.e., circSLC8A1_11, circSLC8A1_12 and circHIPK3_1) (Fig. 2b). In contrast, circSETD3_3, circMB_1 and circCORO1C_1, together with some others (Additional file 12: Figure S7B), appeared to be independently transcribed with their linear parent genes (Fig. 2c). Taken together, by integrating results from multiple studies, our analyses define the most abundant circRNAs in the healthy human heart.

\section{Identification of dysregulated circRNAs in DCM}

We next performed differential analysis to identify circRNAs that are dysregulated in DCM. Using a threshold of $p<0.05$ and $\mathrm{FC}>2$, a total of 392 circRNAs, including 101 up-regulated and 291 down-regulated circRNAs, were identified to be differentially expressed in DCM hearts as compared to normal hearts (Fig. 3a and Additional file 10: Table S5). These differentially expressed circRNAs were derived from 290 unique host genes. Pathway analysis revealed that these host genes are significantly overrepresented in pathways associated with diseases of the heart, including arrhythmogenic right ventricular cardiomyopathy (ARVC), hypertrophic cardiomyopathy, and DCM (Fig. 3b). Interestingly, 2 rt-circRNAs originating from SCAF8 and TIAM2, including SCAF8_e4:TIAM2 e1 and SCAF8_e4:TIAM2_e2, were remarkably downregulated in DCM (Fig. 3c). Many of the significantly down- and up-regulated circRNAs were positively 


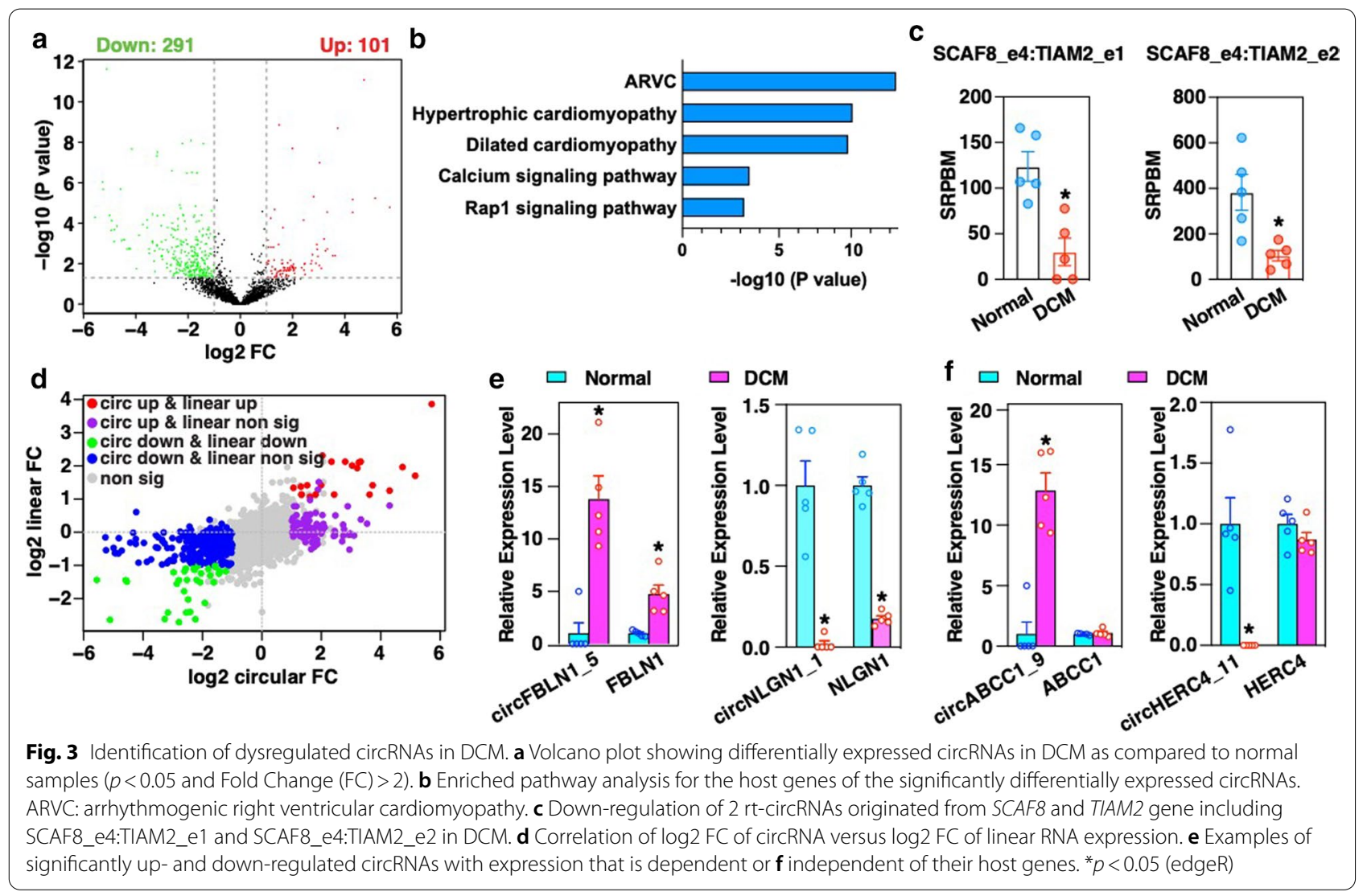

correlated with the expression of their host genes such as circFBLN1_5 with FBLN1 and circNLGN1_1 with $N L G N 1$, respectively (Fig. 3d, e). However, the expression of some circRNAs in DCM was independent of changes in their host gene expression (Fig. 3f). Examples of this discordant relationship included circABCC1_9 and circHERC4_11, which was significantly up- and downregulated in DCM, respectively, while the expression of their host gene mRNAs had no obvious change in DCM (Fig. 3f). Together, these results indicate that dysregulation of circRNAs in DCM tend to originate from heart disease-related gene loci, which include the rt-circRNAs from SCAF8 and TIAM2.

\section{Screening circRNAs for potential as miRNA sponges}

To screen for cardiac circRNAs with the potential to act as miRNA sponges, we analyzed the sequences of each high-confidence circRNAs for the presence of putative binding sites for 50 miRNAs that have established roles in heart disease (Additional file 2: Table S1). In total, 142 circRNAs were predicted to harbor at least 5 predicted binding sites for at least one of 34 heart disease-related miRNAs (Additional file 13: Table S6). Additionally, a total of 16,472 putative targeted genes of these miRNAs were identified, including 598 and 276 up- and down-regulated genes in DCM hearts compared to normal hearts (Additional file 14: Table S7). A circRNA-miRNA-mRNA regulatory network was further constructed based the predicted interactions between circRNAs and miRNAs, as well miRNAs and mRNAs (Fig. 4a). The top 32 circRNAs that contained more than 10 putative binding sites for at least one miRNA are listed in Fig. 4b. Notably, 23 of these are derived from one gene, TTN (Fig. 4b). In particular, circALMS1_6 had the strongest potential to

\footnotetext{
(See figure on next page.)

Fig. 4 Prediction of circRNA potential for sponging miRNAs. a Predicted circRNA-miRNA-circRNA regulatory network. High-abundance circRNAs with at least 5 predicted binding sites (BS) for at least 1 heart disease-related miRNA were included $(n=142)$. $\mathbf{b}$ Heatmap showing the number of BS of circRNAs with at least 10 BS for at least one heart disease-related miRNA. c Schematic diagram of circALMS1_6 which was predicted to have the strongest potential to sponge miR-133. d Schematic diagram of the rt-circRNA, circSCAF8_e4:TIAM2_e2, which was predicted to have potential to sponge multiple heart disease-related miRNAs. Colored rectangles indicate exons and the numbers within them represent the ID of exons. $\mathbf{e}$ Expression of miR-17 target genes in normal vs DCM human hearts. ${ }^{*} p<0.05$ (edgeR)
} 
a Predicted circRNAs-miRNAs-mRNAs regulatory network

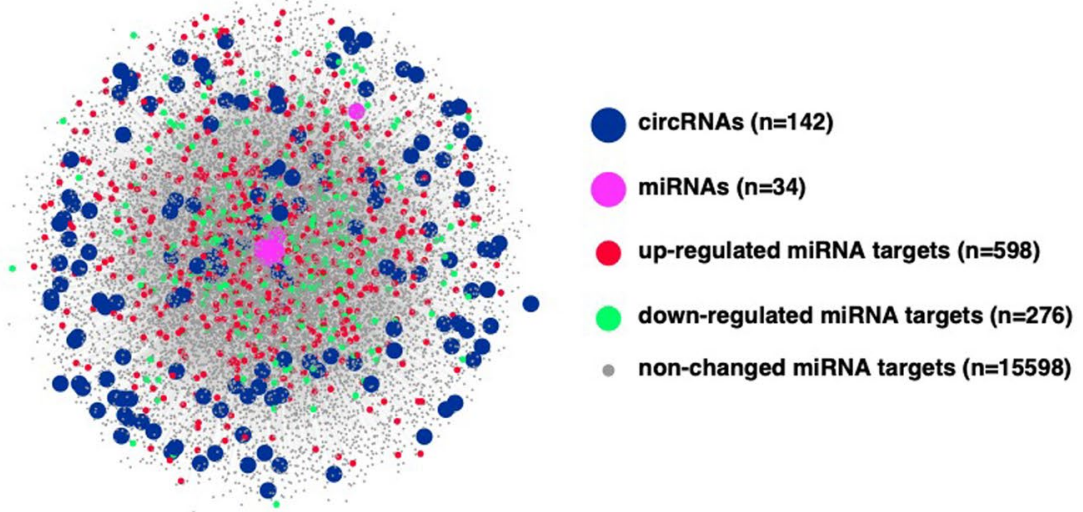

b circRNAs with $>10$ predicted binding sites for heart-associated miRNAs

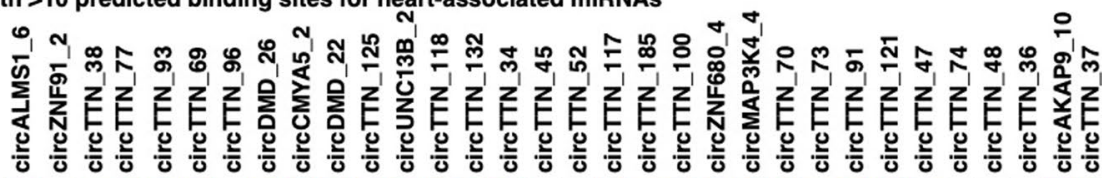

miR-133a-5p

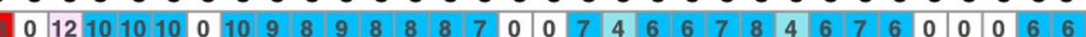

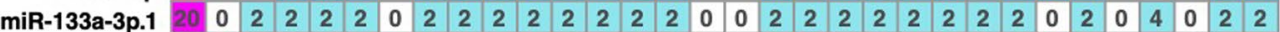

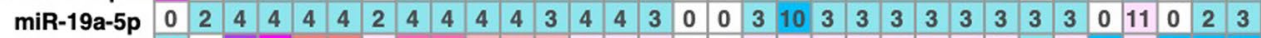

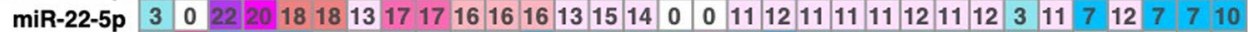
\begin{tabular}{l|l|lllll|l|l|l|l|l|l|l|l|l|l|l|l|l|l|l|l|l|l|l|l|l|l|l|l|l|l|} 
miR-23a-3p & 3 & 24 & 6 & 6 & 6 & 5 & 0 & 5 & 5 & 5 & 5 & 6 & 5 & 5 & 3 & 2 & 14 & 5 & 9 & 5 & 3 & 3 & 3 & 5 & 5 & 5 & 5 & 0 & 3 & 0 & 3 & 3 \\
\hline
\end{tabular}

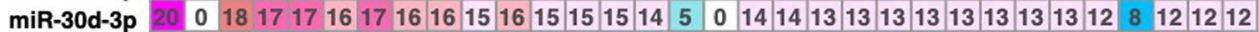
\begin{tabular}{l|l|l|lllllllllllllllllllllllll|l|l|l|ll} 
miR-150-3p & 0 & 0 & 12 & 10 & 7 & 10 & 0 & 10 & 9 & 8 & 9 & 7 & 5 & 8 & 8 & 0 & 0 & 5 & 2 & 5 & 8 & 8 & 9 & 4 & 5 & 3 & 5 & 0 & 2 & 0 & 4 & 7 \\
\hline
\end{tabular}

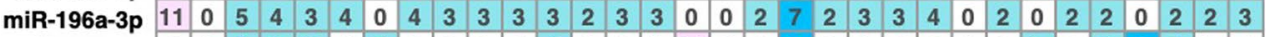

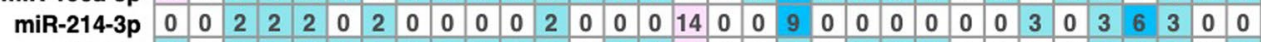
BS numbers \begin{tabular}{lllll|l|l|l|l|l|l|l|l|l|l|l|l|l|l|l|l|l|l|l|l|l|l|l|l|l|l|l|l|}
$\operatorname{miR}-646$ & 3 & 0 & 3 & 3 & 2 & 2 & 4 & 2 & 2 & 2 & 2 & 2 & 0 & 2 & 2 & 11 & 0 & 0 & 5 & 0 & 2 & 2 & 2 & 0 & 0 & 0 & 0 & 2 & 3 & 2 & 0 & 2 \\
\hline
\end{tabular}

\begin{tabular}{|l|}
\hline 28 \\
24 \\
22 \\
20 \\
18 \\
17 \\
16 \\
\hline $11-15$ \\
$6-10$ \\
\hline $2-5$ \\
\hline 0
\end{tabular}

C circALMS1_6, predicted binding sites

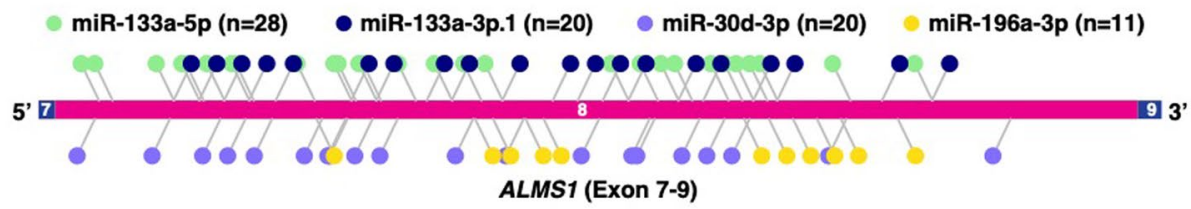

d circSCAF8_e4:TIAM2_e2, predicted binding sites

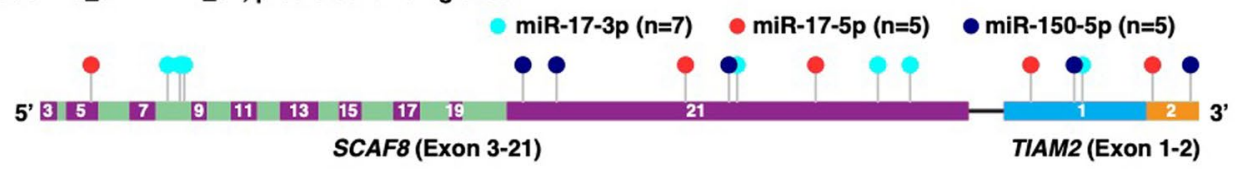

e Expression of miR-17 target genes
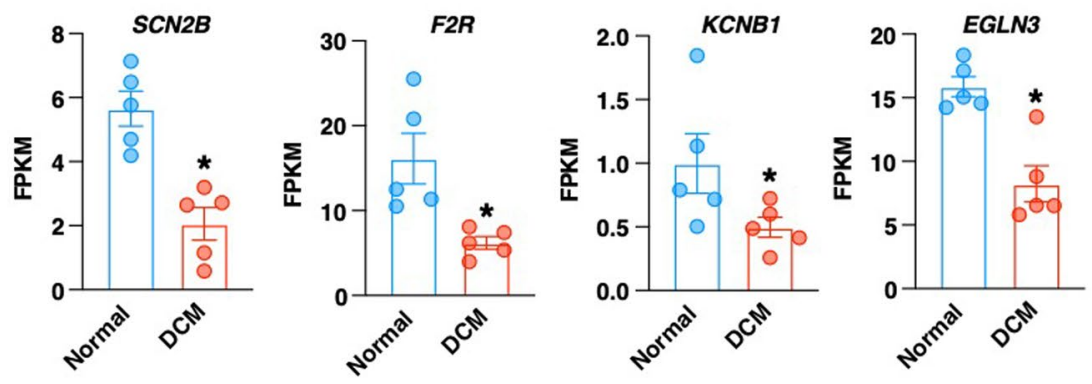
function as miRNA sponge, containing 28 and 20 putative binding sites for miR-133a-5p and miR-133a-3p, respectively (Fig. 4c). Another notable finding is that the rt-circRNA, circSCAF8_e4:TIAM2_e2, was predicted to be capable of sponging multiple miRNAs including miR-150-5p and miR-17 that have been reported to be induced in the rat heart of myocardial ischemia reperfusion injury [50] and in the mouse heart of chronic hypoxia-induced pulmonary hypertension mouse model [51] (Fig. 4d). Interestingly, some of the miR-17 downstream targets, such as SCN2B [52, 53], F2R [54], KCNB1 [55] and EGLN3 [56] that have been implicated in heart dysfunction, were down-regulated in the DCM hearts as revealed by expression analysis of linear RNAs, likely through the unleashed miR-17 that resulted from the down-regulation of circSCAF8_e4:TIAM2_e2 in DCM (Fig. 4e). Collectively, these analyses provide a systematic evaluation of the potential of cardiac circRNAs to sponge miRNAs and provide a resource for studying cardiac miRNA inhibitors.

\section{Experimental validation of identified circRNAs}

Due to lack of availability of human samples, we next sought to validate the conserved circRNAs with mouse orthologs that we identified above using mouse heart samples. We designed divergent primers for the murine orthologs of 6 identified human cardiac circRNAs (Additional file 3: Table S2). These circRNAs were chosen to represent 2 of the most abundant cardiac circRNAs (circEXOC6B_13, circALPK2_2 and circSLC8A_11), 2 of the dysregulated circRNAs (circENAH_4 and circLRP6_4), and 1 non-changed circRNAs between DCM and normal hearts (circN4BP2L2_13). Importantly, these circRNAs have not been experimentally verified by previous studies, with exception of circSLC8A_11 that served as a positive control for circRNA amplification in this study. We performed PCR amplification using mouse heart cDNA as the template, with mouse heart genomic DNA used as a negative control and mouse Yap1 gene primers used as an internal control for the PCR amplification of genomic DNA. All 6 of the selected circRNAs were successfully amplified in heart cDNA but not in genomic DNA (Fig. 5a). Sanger sequencing of the PCR products verified back-spliced junctions (Fig. 5b-g). Taken together, all the circRNAs examined were confirmed to be bona fide, suggesting the reliability of our in silico circRNA identification.

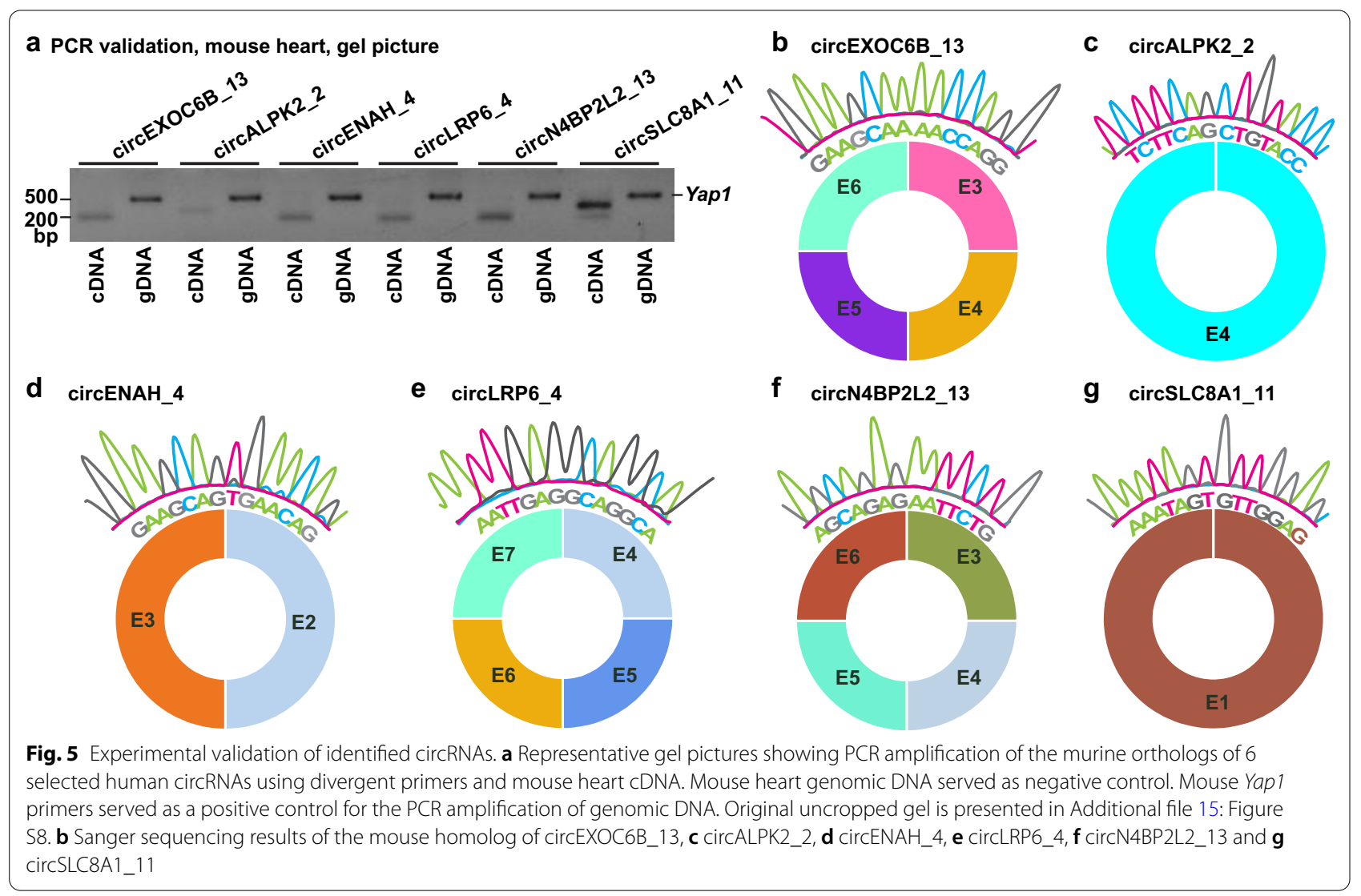




\section{Discussion}

Herein, we have analyzed rRNA-depleted RNA sequencing datasets from 10 human hearts. By integrating circRNAs that were previously identified in human, mouse and rat hearts [26-29, 40, 41, 43, 49], we have systematically interrogated the global landscape of circRNAs in human heart and have revealed the most abundant circRNAs in healthy hearts, and those that are dysregulated in DCM hearts. Furthermore, we have investigated the potential of human cardiac circRNAs to act as sponges for heart disease-related miRNAs and have generated a list of rtcircRNAs expressed in human heart. Our study adds new information to the previous annotated circRNAs and provides a valuable resource for further functional study of circRNAs in the human heart.

Three earlier studies found that circRNAs are expressed in the human heart and identified a large number of circRNAs [27-29]. The number of circRNAs identified in our study $(n=20,214)$ is larger than that of previous studies. This could be partially explained by the differences in the genetic background of the human subjects, the algorithms employed and the thresholds used for detecting and filtering circRNAs, and sample size. When comparing the degree of overlap of circRNAs identified in previous studies, congruency was lower than expected despite the use of the same tissue (heart) and species (human). For example, only about half of the circRNAs reported by the three previous studies are in common [27-29] (Fig. 1h). This observation suggests that either the expression pattern of circRNAs varies significantly among different individuals, or that the identified circRNAs in the limited samples were artifactual, which underscores the need to identify a list of reliable cardiac circRNAs by integrating multiple independent studies. Our comparative analysis revealed a total 16,304 cardiac circRNAs that are reproducible in at least one of previous studies (Fig. 1h and Additional file 5: Table S3), which represent a bona fide category of cardiac circRNAs that can be functionally explored in the future.

Our analysis revealed that a significant number of cardiac circRNAs are derived from two different genes, which were also reported by previous studies of circRNAs in heart [27, 29] and brain [16, 36, 46]. Due to the fact that many such circRNAs are derived from neighboring genes with highly homologous sequences, a common practice in circRNA detection is to discard circRNAs from multiple genes [28, 46]. However, a recent breakthrough study showed that a significant portion of circRNAs indeed originate from the exons of two adjacent genes on the same strand. These circRNA are referred to as rt-circRNAs [30] and suggests that circRNAs originated from different genes should not simply be dismissed as false positives, especially in studies with a major focus on circRNA discovery. One striking observation of this study is that a number of circRNAs are identified to be produced from the exons of $M Y H 6$ and $M Y H 7$, which were also frequently observed, and display high abundance, in hearts of human, mouse and rat by two previous studies (Additional file 7: Figure S3A-D) $[27,29]$, albeit using different algorithms and cohorts for circRNA detection. This could be particularly interesting if the circRNAs derived from $M Y H 6$ and $M Y H 7$ are bona fide, because these two genes play important roles in healthy and diseased hearts [57]. However, in-depth examination of sequences reveals that these circRNAs are derived from highly homologous region of $M Y H 6$ and $M Y H 7$, suggesting that these circRNAs are artifacts due to the read misalignment as described by previous studies $[28,46]$. Therefore, cautions should be taken when annotating circRNAs from multiple genes. Nevertheless, after filtering out additional circRNAs from the homologous regions of two nearby genes, we identified a total of 110 potentially bona fide rt-circRNAs (Additional file 6: Table S4). Especially, SCAF8 and TIAM2 gene produce the largest number of rt-circRNAs (Fig. 1c). Notably, all of the identified putative rt-circRNAs in human heart are not conserved in mouse or rat, suggesting that they are human-specific and recently evolved novel non-coding molecules. Future efforts are needed to validate these rtcircRNAs in greater detail and explore their functional roles in the heart.

Our analysis yielded a list of the top 30 most abundant circRNAs expressed in normal hearts (Fig. 2a). All of the identified top circRNAs, except for circSPHKAP_1 and circRMST_3, are within the top 100 most abundant circRNAs in human or mouse hearts as revealed by at least one previous study (Fig. 2a) [27, 29], suggesting a high degree of fidelity of this top circRNA list. In previous studies, discordant findings have been observed for some of these top circRNAs with regard to their presence and abundance $[27,29]$. For instance, circTULP4_1 is absent in the study of Tan et al. [27] but is abundantly expressed in human and mouse heart. Our cross-reference analysis of multiple independent studies helps to reduce confusion over the importance of the identified circRNAs. The function of one of the most abundant circRNAs, circSLC8A1_11, in heart function has been assessed by a recent study [58]. CircHIPK3_1, formed from the circularization of the second exon of HIPK3, is another conserved circRNA that is highly expressed in both human and murine heart. An earlier study has shown that circHIPK3_1 plays a critical role in promoting retinal vascular dysfunction in diabetes mellitus [59]. In future studies, it will be interesting to explore the function of circHIPK3_1, as well as some of the other top candidates we have identified such as circTULP4_1 (Fig. 2a), in 
maintaining cardiac hemostasis. Furthermore, half of the most abundant human circRNAs are conserved in mouse and/or rat. However, only several of these, including circSLC8A1_11, circHIPK3_1, circQKI_4 and circTULP4_1 are observed to be highly expressed in murine heart as well, indicating differences in the circRNA landscape between humans and rodents. In addition, the expression levels of the three human-specific circRNAs including circMB_1, circCORO1C_1 and circSETD3_3 tends to be independent of their host genes, suggesting potentially important roles of these circRNAs in human heart.

Differential analysis revealed that most of the aberrant circRNAs were down-regulated in DCM as compared to normal hearts (Fig. 3a), a feature that is similar to observations in cancer [30, 60]. In cancer, decreased expression of circRNAs was speculated to result from the dilution of circRNA upon cancer cell division [30, 60]. However, this mechanism cannot account for the reduced expression in DCM hearts due to the fact that adult cardiac myocytes have a very limited replicative capacity and extensive cell death leads to a reduction in myocyte number in DCM [61]. Another interesting finding is that many of the abundant circRNAs originate from already-established cardiovascular disease-related gene loci, as indicated by the results of pathway analysis of their host genes (Fig. 3b). For example, four circRNAs generated from the TTN locus, including circTTN_34, circTTN_52, circTTN_70 and circTTN_132 were significantly downregulated in DCM as compared to normal hearts (Additional file 10: Table S5). These data are consistent with an earlier study that reported on the dysregulation of circRNAs synthesized from the TTN gene in DCM [62]. The TTN gene encodes the protein titin, which is the largest known protein expressed in the heart. The TTN gene was also shown to generate the largest number of circRNAs in the heart (Additional file 8: Figure S4A). Another interesting gene which generated two significantly downregulated circRNAs (circRYR2_71 and circRYR2_95) in DCM, was the RYR2 gene. RYR2 is the major $\mathrm{Ca}^{2+}$ release channel on the sarcoplasmic reticulum and plays a critical role in regulating excitation-contraction coupling and $\mathrm{Ca}^{2+}$ homeostasis in the heart [63]. Sequence variations of these two genes that result in altered amino acid sequence and disruption of protein structure and function, have been linked to DCM $[64,65]$. There was no evidence to support the presence of mutations in TTN and RYR2 genes in the 5 DCM patients analyzed. Furthermore, RNA-seq analysis revealed that their expression did not change at the mRNA level as compared to controls. Thus the downregulation of circRNAs observed from the TTN and RYR2 genes in DCM likely represent a novel mechanism that awaits further identification. We observed that multiple circRNAs were differentially expressed in DCM, with no obvious change in the parent gene expression (Fig. 3d and f), confirming previous findings that the expression of circRNAs can be independent of their parent genes [66].

One of the best documented functions of circRNAs is to sponge miRNAs, which is similar to linear long noncoding RNAs [67, 68]. Furthermore, the regulatory role of circRNAs via the circRNA-miRNA-mRNA axis has been increasingly implicated in human diseases [69]. In addition, the relatively higher stability of circRNAs compared to linear mRNAs due to the lack of free $5^{\prime}$ and $3^{\prime}$ ends allow them to be ideal molecular sponges for miRNAs. Our results in predicting the ability of circRNAs to bind heart disease-related miRNAs provides a guideline for further exploration of the regulatory roles of cardiac circRNAs (Fig. 4a, Additional file 13: Table S6, and Additional file 14: Table S7). Notably, circRNAs produced from the TTN gene show a strong potential to bind miRNAs, suggesting that the function of the cardiac TTN gene may have added complexity. In addition, circALMS1_6 has strong potential to sponge miR133, a well-known miRNA that is abundantly expressed in skeletal muscle and cardiomyocytes [70]. Given the important roles of miR-133 in regulating cardiac hypertrophy, it will be interesting to determine whether circALMS1_6 affects cardiac hemostasis via inhibition of miRNAs. Furthermore, we identified a rt-circRNA, circSCAF8_e4:TIAM2_e2, with the potential to bind multiple miRNAs including miR-17 that have been shown to be induced in diseased hearts $[50,51]$ (Fig. 4d). In DCM hearts, the expression of circSCAF8_e4:TIAM2_ $\mathrm{e} 2$ is decreased (Fig. 3c). It is therefore possible that the down-regulation of this rt-circRNA contributes to the up-regulation of miR-17 via releasing the sequestration of miR-17 interacted with, thereby inhibiting the downstream targets of miR-17. In support of this hypothesis, several miR-17 targets with known cardiac function are down-regulated in DCM hearts (Fig. 4e). One interesting target is $S C N 2 B$ that encodes voltage-gated sodium channel $\beta 2$-subunits. The sequence variations of $S C N 2 B$ have been associated with human cardiac arrhythmias [53] and genetic deletion of $S c n 2 b$ in mice leads to ventricular and atrial arrhythmias [52]. Our study suggests a possible regulatory role of circSCAF8_e4:TIAM2_e2-miR-17$S C N 2 B$ axis in heart and expands our understanding of the function of the newly discovered rt-circRNAs.

\section{Conclusions}

In conclusion, our study provides a list of putative key circRNAs that are expressed in normal and DCM human hearts. These circRNAs we identified are helpful to guide future functional studies. Given their abundance and multiple functional roles, circRNAs, including 
rt-circRNAs, are expected to be a group of emerging players in the regulation of human heart diseases.

\section{Supplementary information}

Supplementary information accompanies this paper at https://doi. org/10.1186/s12920-020-00817-7.

Additional file 1. Figure S1. Flowchart for de novo identification of circRNAs in human heart.

Additional file 2. Table S1. Information of DCM-related miRNAs used for analysis of sponge potential. The seed sequence of each miRNA was obtained from TargetScan database. PMID is the PubMed number for studies which reported the miRNAs.

Additional file 3. Table S2. Primers used for validation of the mouse ortholog of selected human circRNAs. Mouse Yap1 primers were used as internal control for PCR reaction using genomic DNA.

Additional file 4. Figure S2. Validation of the public RNA-seq dataset. (A) Principal Component Analysis (PCA) of the 10 samples (5 normal and 5 DCM hearts) using expression of genes with raw count $>10$ in at least 5 samples. (B) Expression of $\boldsymbol{N P P A}$ in normal and DCM hearts from the RNAseq dataset. PFKM: Fragments Per Kilobase of exon per Million fragments mapped. ${ }^{*} p<0.05$ (edgeR).

Additional file 5. Table S3. Information of the 20,214 circRNAs identified in this study. Chromosome coordinates are in hg38 annotation.

Additional file 6. Table S4. List of circRNAs originated from two different genes in 10 human heart samples. Chromosome coordinates are in hg38 annotation. In the last column, "yes" indicates that the circRNA is from two neighboring genes with highly similar sequence, and "no" indicates no sequence similarity was observed and the circRNA is likely a bona fide rt-circRNA.

Additional file 7. Figure S3. Analysis of artifactual circRNAs from MYH6 and MYH7 gene. (A) Schematic diagram showing a number of rt-circRNAs originate from $\mathbf{M Y H \boldsymbol { 6 }}$ and $\mathbf{M Y H 7}$ genes in human heart identified in this study, study of Tan et al. [27] and Werfel et al. [29], and in (B) mouse heart identified in this study and study of Werfel et al. [29], as well as in (C) rat heart revealed in the study of Werfel et al. [29]. Arrows point to the transcriptional directions of $\mathbf{M Y H} \mathbf{6}$ and $\boldsymbol{M} \boldsymbol{Y H} \mathbf{7}$ genes. (D) Heatmap illustrates the rank of the average expression across all studied samples for circRNAs produced from $\mathbf{M Y H 6}$ and $\boldsymbol{M Y H 7}$ genes in this study, and in human, mouse and rat hearts as identified by Tan et al. [27] and Werfel et al. [29]. The numbers in the colored boxes indicate the rank of the circRNAs within the indicated species. (E) Representative example of circMYH6_ e35:MYH7_e37 that is likely artifact. The upper schematic diagram illustrating the formation of circMYH6_e35:MYH7_e37 due to read misalignment. The exon 35 of $\boldsymbol{M Y H \boldsymbol { 6 }}$ (red, the donor exon) and exon 36 of $\boldsymbol{M Y H 7}$ (yellow, upstream of the acceptor exon) are homologous. The exon 36 of $\mathbf{M Y H 6}$ (green, downstream of the donor exon) and exon 37 of $\mathbf{M Y H 7}$ (blue, the acceptor exon) are highly homologous. Considering a chimeric sequencing read that is supposedly to be aligned back to exon 35 (red line without arrow) and 36 (green line with arrow) of $\mathbf{M Y H 6}$, due to the high sequence similarity, the down-stream part of the read (green line with arrow) may be misaligned to exon 37 of $\boldsymbol{M Y H 7}$ (blue line with arrow), leading to the artifactual identification of back-spliced junction, and versa vice. Dashed lines indicate splicing junctions. The bottom panel shows the sequence alignment of linear MYH6_e35:MYH6_e36, MYH7_e36:MYH7_e37, and the predicted sequence of the circular MYYH6_e35:MȲH7_e37.

Additional file 8. Figure S4. Relationship between numbers of circRNAs and exons of their host genes. (A) Schematic diagram of all the circRNAs originated from $\boldsymbol{T T N}$ and (B) $\boldsymbol{R} Y \boldsymbol{R} \mathbf{2}$ gene. Arrows point to the transcriptional directions of $\boldsymbol{T} \boldsymbol{T} \boldsymbol{N}$ and $\boldsymbol{R} \boldsymbol{Y} \boldsymbol{R} \mathbf{2}$ genes. (C) The numbers of circRNAs increased proportionally with the numbers of exons per gene (binned to 10). (D) Distribution of exon numbers to produce circRNAs.

Additional file 9. Figure S5. Comparison of circRNAs with 2 unique back-spliced reads observed only once in this study with human circRNAs reported by 3 previous studies and 4 circRNA databases. (A) Four-set venn diagram showing the number of over-lapping circRNAs between the 12,099 circRNAs with 2 unique back-spliced reads observed only in one sample in this study, and human cardiac circRNAs reported by 3 previous studies. (B) Five-set venn diagram showing the number of over-lapping circRNAs between the 12,099 circRNAs with 2 unique back-spliced reads observed only in one sample in this study, and human circRNAs reported by 4 circRNA databases. (C) Three-set venn diagram showing the number of over-lapping circRNAs between the 12,099 circRNAs with 2 unique back-spliced reads observed only in one sample in this study, and 24,256 and 263,738 non-redundant circRNAs obtained by merging circRNAs reported by study of Werfel et al. ( $n=16,427)$, Tan et al. ( $n=15,303)$, and Van et al. $(n=8878)$, as well as 4 circRNA datasets including circBase $(n=$ 91,986), circBank ( $n=140,331)$, circRNADB ( $n=32,883$ ), and CIRCpedia ( $n$ $=183,943)$.

Additional file 10. Table S5. List of high-confidence and significantly dysregulated circRNAs in DCM compared to normal heart samples. Chromosome coordinates are in hg38 annotation. The threshold for significance was $p<0.05$ and fold change $>2$.

Additional file 11. Figure S6. Comparison of the 2461 high-abundance circRNAs with reported human, mouse and rat circRNAs. (A) Three-set venn diagram showing the number of over-lapping circRNAs between the 2461 high-abundance circRNAs identified in this study, and 24,256 and 263,738 non-redundant circRNAs obtained by merging circRNAs reported by study of Werfel et al. ( $n=16,427)$, Tan et al. $(n=15,303)$, and Van et al. ( $n=8,878)$, as well as 4 circRNA datasets including circBase $(n=91,986)$, circBank ( $n=140,331)$, circRNADB $(n=32,883)$, and CIRCpedia ( $n=$ 183,943). (B) Three-set venn diagram showing the number of over-lapping circRNAs between the 2461 high-abundance circRNAs identified in this study, and 61,174 and 20,450 reported mouse and rat circRNAs. The 61,174 non-redundant mouse circRNAs were obtained by merging circRNAs reported by study of Werfel et al. ( $n=9474)$, Jakobi et al. $(n=561)$, and Tan et al. ( $n=2632)$, as well as 2 circRNA databases including circBase ( $n$ $=1756$ ) and CIRCpedia V2 ( $n=54,274)$. The 20,450 non-redundant rat circRNAs were obtained by merging circRNAs reported by study of Werfel et al. $(n=12,256)$ and CIRCpedia V2 $(n=17,083)$.

Additional file 12. Figure S7. Correlation of expression between 24 the most abundant circRNAs and their host genes. (A) CircRNAs with expression significantly correlated with the expression of host genes (Pearson correlation, $p<0.05)$. (B) CircRNAs with expression independent of their host genes (Pearson correlation, $p>0.05$ ).

Additional file 13. Table S6. CircRNAs with potential to sponge heart disease-related miRNAs. CircRNAs with at least 5 predicted binding sites for at least one miRNA were listed.

Additional file 14. Table S7. Predicted targets of heart disease-related miRNAs. The targeted genes of each miRNA were obtained from TargetScan (http://www.targetscan.org/vert_72/). The significance of changes of targeted genes in DCM compared to normal samples were determined by DESeq 2 with threshold of fold change $>2$ and FDR $<0.05$. A total of 937 and 480 genes were identified to be significantly up- and down-regulated in DCM, respectively.

Additional file 15. Figure S8. Original uncropped PCR gel picture (related to Fig. 5a)

\section{Abbreviations}

circRNA: Circular RNA; rt-circRNA: Read-through circular RNA; HF: Heart failure; DCM: Dilated cardiomyopathy; PCA: Principle component analysis; UTR: Untranslated region; CDS: Coding sequence; FC: Fold change; ARVC: Arrhythmogenic right ventricular cardiomyopathy; FPKM: Fragments per kilobase of exon per million fragments mapped; SRPBM: Spliced reads per billion mapped reads.

\section{Acknowledgements}

We gratefully thank all members at the J.Z. lab for their stimulating discussions of this work. 


\section{Authors' contributions}

J.Z. and K.D. conceived and designed the study. K.D. performed the bioinformatics analysis. K.D. and X.H. performed experimental validation of circRNAs. K.D. wrote the manuscript. H.S., D.F. and J.Z. edited the manuscript. All authors have read and approved the manuscript.

\section{Funding}

The work at the J.Z. laboratory is supported by a grant (R01HL149995) from the National Heart, Lung, and Blood Institute, NIH. J.Z. is a recipient of Established Investigator Award (17EIA33460468) and Transformational Project Award (19TPA34910181) from the American Heart Association. K.D. is supported by a postdoctoral fellowship (19POST34450071) from the American Heart Association. The funders provided the financial support to this research, but had no role in the design of the study, analysis, interpretations of the data and in writing the manuscript.

\section{Availability of data and materials}

The RNA-seq data used in this study was downloaded from NCBI database under Accession Number SRP108571.

\section{Ethics approval and consent to participate}

Not applicable.

\section{Consent for publication}

Not applicable.

\section{Competing interests}

The authors declare that they have no competing interests.

\section{Author details}

${ }^{1}$ Department of Pharmacology and Toxicology, Medical College of Georgia, Augusta University, 1459 Laney Walker Blvd, Augusta, GA 30912, USA. ${ }^{2}$ Vascular Biology Center, Medical College of Georgia, Augusta University, Augusta, GA 30912, USA.

Received: 27 July 2020 Accepted: 28 October 2020

Published online: 07 November 2020

\section{References}

1. Ziaeian B, Fonarow GC. Epidemiology and aetiology of heart failure. Nat Rev Cardiol. 2016;13(6):368-78.

2. Lakdawala NK, Winterfield JR, Funke BH. Dilated cardiomyopathy. Circ Arrhythm Electrophysiol. 2013;6(1):228-37.

3. Bruneau BG. The developmental genetics of congenital heart disease. Nature. 2008;451(7181):943-8.

4. Bozkurt B, Colvin M, Cook J, Cooper LT, Deswal A, Fonarow GC, Francis GS, Lenihan D, Lewis EF, McNamara DM, et al. Current diagnostic and treatment strategies for specific dilated cardiomyopathies: a scientific statement from the American Heart Association. Circulation. 2016;134(23):e579-646.

5. McNally EM, Mestroni L. Dilated cardiomyopathy: genetic determinants and mechanisms. Circ Res. 2017;121(7):731-48.

6. Pugh TJ, Kelly MA, Gowrisankar S, Hynes E, Seidman MA, Baxter SM, Bowser M, Harrison B, Aaron D, Mahanta LM, et al. The landscape of genetic variation in dilated cardiomyopathy as surveyed by clinical DNA sequencing. Genet Med. 2014;16(8):601-8.

7. Sweet ME, Cocciolo A, Slavov D, Jones KL, Sweet JR, Graw SL, Reece TB, Ambardekar AV, Bristow MR, Mestroni L, et al. Transcriptome analysis of human heart failure reveals dysregulated cell adhesion in dilated cardiomyopathy and activated immune pathways in ischemic heart failure. BMC Genom. 2018;19(1):812.

8. Molina-Navarro MM, Trivino JC, Martinez-Dolz L, Lago F, Gonzalez-Juanatey JR, Portoles M, Rivera M. Functional networks of nucleocytoplasmic transport-related genes differentiate ischemic and dilated cardiomyopathies: a new therapeutic opportunity. PLoS ONE. 2014;9(8):e104709.

9. Barth AS, Kuner R, Buness A, Ruschhaupt M, Merk S, Zwermann L, Kaab S, Kreuzer E, Steinbeck G, Mansmann U, et al. Identification of a common gene expression signature in dilated cardiomyopathy across independent microarray studies. J Am Coll Cardiol. 2006;48(8):1610-7.

10. Qiu Z, Ye B, Yin L, Chen W, Xu Y, Chen X. Downregulation of AC061961.2, LING01-AS1, and RP11-13E1.5 is associated with dilated cardiomyopathy progression. J Cell Physiol. 2019;234(4):4460-71.

11. Zhang X, Nie X, Yuan S, Li H, Fan J, Li C, Sun Y, Zhao Y, Hou H, Wang DW, et al. Circulating long non-coding RNA ENST00000507296 Is a Prognostic indicator in patients with dilated cardiomyopathy. Mol Ther Nucleic Acids. 2019;16:82-90.

12. Satoh M, Minami Y, Takahashi Y, Tabuchi T, Nakamura M. Expression of microRNA-208 is associated with adverse clinical outcomes in human dilated cardiomyopathy. J Card Fail. 2010;16(5):404-10.

13. Satoh M, Minami Y, Takahashi Y, Tabuchi T, Nakamura M. A cellular microRNA, let-7i, is a novel biomarker for clinical outcome in patients with dilated cardiomyopathy. J Card Fail. 2011;17(11):923-9.

14. Onrat ST, Onrat E, Ercan Onay E, Yalim Z, Avsar A. The genetic determination of the differentiation between ischemic dilated cardiomyopathy and idiopathic dilated cardiomyopathy. Genet Test Mol Biomark. 2018;22(11):644-51.

15. Memczak S, Papavasileiou P, Peters O, Rajewsky N. Identification and characterization of circular RNAs As a new class of putative biomarkers in human blood. PLoS ONE. 2015;10(10):e0141214.

16. Rybak-Wolf A, Stottmeister C, Glazar P, Jens M, Pino N, Giusti S, Hanan M, Behm M, Bartok O, Ashwal-Fluss R, et al. Circular RNAs in the Mammalian brain are highly abundant, conserved, and dynamically expressed. Mol Cell. 2015;58(5):870-85.

17. Qu S, Yang X, Li X, Wang J, Gao Y, Shang R, Sun W, Dou K, Li H. Circular RNA: a new star of noncoding RNAs. Cancer Lett. 2015;365(2):141-8.

18. Legnini I, Di Timoteo G, Rossi F, Morlando M, Briganti F, Sthandier O, Fatica A, Santini T, Andronache A, Wade M, et al. Circ-ZNF609 is a circular RNA that can be translated and functions in myogenesis. Mol Cell. 2017;66(1):22-37.

19. Hall IF, Climent M, Quintavalle M, Farina FM, Schorn T, Zani S, Carullo P, Kunderfranco P, Civilini E, Condorelli G, et al. Circ_Lrp6, a circular RNA enriched in vascular smooth muscle cells, acts as a sponge regulating miRNA-145 function. Circ Res. 2019;124(4):498-510.

20. Chen J, Cui L, Yuan J, Zhang Y, Sang H. Circular RNA WDR77 target FGF-2 to regulate vascular smooth muscle cells proliferation and migration by sponging miR-124. Biochem Biophys Res Commun. 2017:494(1-2):126-32.

21. Holdt LM, Stahringer A, Sass K, Pichler G, Kulak NA, Wilfert W, Kohlmaier A, Herbst A, Northoff BH, Nicolaou A, et al. Circular non-coding RNA ANRIL modulates ribosomal RNA maturation and atherosclerosis in humans. Nat Commun. 2016;7:12429.

22. Burd CE, Jeck WR, Liu Y, Sanoff HK, Wang Z, Sharpless NE. Expression of linear and novel circular forms of an INK4/ARF-associated non-coding RNA correlates with atherosclerosis risk. PLoS Genet. 2010;6(12):e1001233.

23. Guarnerio J, Bezzi M, Jeong JC, Paffenholz SV, Berry K, Naldini MM, LoCoco F, Tay Y, Beck AH, Pandolfi PP. Oncogenic role of fusion-circRNAs derived from cancer-associated chromosomal translocations. Cell. 2016;165(2):289-302

24. Chen S, Huang V, Xu X, Livingstone J, Soares F, Jeon J, Zeng Y, Hua JT, Petricca J, Guo H, et al. Widespread and functional RNA circularization in localized prostate cancer. Cell. 2019;176(4):831-43.

25. Hu W, Bi ZY, Chen ZL, Liu C, Li LL, Zhang F, Zhou Q, Zhu W, Song YY, Zhan BT, et al. Emerging landscape of circular RNAs in lung cancer. Cancer Lett. 2018:427:18-27.

26. Jakobi T, Czaja-Hasse LF, Reinhardt R, Dieterich C. Profiling and validation of the circular RNA repertoire in adult murine hearts. Genom Proteom Bioinform. 2016;14(4):216-23.

27. Tan WL, Lim BT, Anene-Nzelu CG, Ackers-Johnson M, Dashi A, See K, Tiang Z, Lee DP, Chua WW, Luu TD, et al. A landscape of circular RNA expression in the human heart. Cardiovasc Res. 2017;113(3):298-309.

28. van Heesch S, Witte F, Schneider-Lunitz V, Schulz JF, Adami E, Faber AB, Kirchner M, Maatz H, Blachut S, Sandmann CL, et al. The translational landscape of the human heart. Cell. 2019;178(1):242-60.

29. Werfel S, Nothjunge S, Schwarzmayr T, Strom TM, Meitinger T, Engelhardt S. Characterization of circular RNAs in human, mouse and rat hearts. J Mol Cell Cardiol. 2016;98:103-7. 
30. Vo JN, Cieslik M, Zhang Y, Shukla S, Xiao L, Zhang Y, Wu YM, Dhanasekaran SM, Engelke CG, Cao X, et al. The landscape of circular RNA in cancer. Cell. 2019;176(4):869-81.

31. Bolger AM, Lohse M, Usadel B. Trimmomatic: a flexible trimmer for Illumina sequence data. Bioinformatics. 2014;30(15):2114-20.

32. Langmead B, Salzberg SL. Fast gapped-read alignment with Bowtie 2. Nat Methods. 2012;9(4):357-9.

33. Anders S, Pyl PT, Huber W. HTSeq: a python framework to work with highthroughput sequencing data. Bioinformatics. 2015;31(2):166-9.

34. Mortazavi A, Williams BA, McCue K, Schaeffer L, Wold B. Mapping and quantifying mammalian transcriptomes by RNA-Seq. Nat Methods. 2008;5(7):621-8.

35. Love MI, Huber W, Anders S. Moderated estimation of fold change and dispersion for RNA-seq data with DESeq2. Genome Biol. 2014;15(12):550.

36. Memczak S, Jens M, Elefsinioti A, Torti F, Krueger J, Rybak A, Maier L, Mackowiak SD, Gregersen LH, Munschauer M, et al. Circular RNAs are a large class of animal RNAs with regulatory potency. Nature. 2013;495(7441):333-8.

37. Li H, Handsaker B, Wysoker A, Fennell T, Ruan J, Homer N, Marth G, Abecasis $\mathrm{G}$, Durbin R. Genome project data processing $\mathrm{S}$ : the sequence alignment/map format and SAMtools. Bioinformatics. 2009;25(16):2078-9.

38. Robinson MD, McCarthy DJ, Smyth GK. edgeR: a bioconductor package for differential expression analysis of digital gene expression data. Bioinformatics. 2010;26(1):139-40.

39. Zhou Y, Zhou B, Pache L, Chang M, Khodabakhshi AH, Tanaseichuk O, Benner C, Chanda SK. Metascape provides a biologist-oriented resource for the analysis of systems-level datasets. Nat Commun. 2019;10(1):1523.

40. Glazar P, Papavasileiou P, Rajewsky N. circBase: a database for circular RNAs. RNA. 2014;20(11):1666-70,

41. Liu M, Wang Q, Shen J, Yang BB, Ding XM. Circbank: a comprehensive database for circRNA with standard nomenclature. RNA Biol. 2019;16(7):899-905.

42. Ye J, Wang L, Li S, Zhang Q, Zhang Q, Tang W, Wang K, Song K, Sablok G, Sun $X$, et al. AtCircDB: a tissue-specific database for Arabidopsis circular RNAs. Brief Bioinform. 2019;20(1):58-65.

43. Dong R, Ma XK, Li GW, Yang L. CIRCpedia v2: an updated database for comprehensive circular RNA annotation and expression comparison. Genom Proteom Bioinf. 2018;16(4):226-33.

44. Hansen TB, Veno MT, Damgaard CK, Kjems J. Comparison of circular RNA prediction tools. Nucleic Acids Res. 2016;44(6):e58.

45. Song W, Wang $\mathrm{H}, \mathrm{Wu} \mathrm{Q}$. Atrial natriuretic peptide in cardiovascular biology and disease (NPPA). Gene. 2015;569(1):1-6.

46. Gruner H, Cortes-Lopez M, Cooper DA, Bauer M, Miura P. CircRNA accumulation in the aging mouse brain. Sci Rep. 2016;6:38907.

47. Salzman J, Gawad C, Wang PL, Lacayo N, Brown PO. Circular RNAs are the predominant transcript isoform from hundreds of human genes in diverse cell types. PLoS ONE. 2012;7(2):e30733.

48. Westholm JO, Miura P, Olson S, Shenker S, Joseph B, Sanfilippo P, Celniker SE, Graveley BR, Lai EC. Genome-wide analysis of drosophila circular RNAs reveals their structural and sequence properties and age-dependent neural accumulation. Cell Rep. 2014;9(5):1966-80.

49. Chen XP, Han P, Zhou T, Guo XJ, Song XF, Li Y. circRNADb: A comprehensive database for human circular RNAs with protein-coding annotations. Sci Rep-Uk 2016, 6.

50. Du W, Pan Z, Chen X, Wang L, Zhang Y, Li S, Liang H, Xu C, Zhang Y, Wu $Y$, et al. By targeting Stat3 microRNA-17-5p promotes cardiomyocyte apoptosis in response to ischemia followed by reperfusion. Cell Physiol Biochem. 2014;34(3):955-65.

51. Pullamsetti SS, Doebele C, Fischer A, Savai R, Kojonazarov B, Dahal BK, Ghofrani HA, Weissmann N, Grimminger F, Bonauer A, et al. Inhibition of microRNA-17 improves lung and heart function in experimental pulmonary hypertension. Am J Respir Crit Care Med. 2012;185(4):409-19.

52. Bao Y, Willis BC, Frasier CR, Lopez-Santiago LF, Lin X, Ramos-Mondragon R, Auerbach DS, Chen C, Wang Z, Anumonwo J et al. Scn2b deletion in mice results in ventricular and atrial arrhythmias. Circ Arrhythm Electrophysiol. 2016, 9(12).
53. Watanabe H, Darbar D, Kaiser DW, Jiramongkolchai K, Chopra S, Donahue BS, Kannankeril PJ, Roden DM. Mutations in sodium channel beta1- and beta2-subunits associated with atrial fibrillation. Circ Arrhythm Electrophysiol. 2009;2(3):268-75.

54. Gigante B, Vikstrom M, Meuzelaar LS, Chernogubova E, Silveira A, Hooft FV, Hamsten A, de Faire U. Variants in the coagulation factor 2 receptor (F2R) gene influence the risk of myocardial infarction in men through an interaction with interleukin 6 serum levels. Thromb Haemost. 2009:101(5):943-53.

55. Arnett DK, Li N, Tang W, Rao DC, Devereux RB, Claas SA, Kraemer R, Broeckel U. Genome-wide association study identifies single-nucleotide polymorphism in KCNB1 associated with left ventricular mass in humans: the HyperGEN Study. BMC Med Genet. 2009;10:43.

56. Frank D, Gantenberg J, Boomgaarden I, Kuhn C, Will R, Jarr KU, Eden M, Kramer K, Luedde M, Mairbaurl H, et al. MicroRNA-20a inhibits stressinduced cardiomyocyte apoptosis involving its novel target Egln3/PHD3. J Mol Cell Cardiol. 2012;52(3):711-7.

57. Hill JA, Olson EN. Cardiac plasticity. N Engl J Med. 2008;358(13):1370-80.

58. Lim TB, Aliwarga E, Luu TDA, Li YP, Ng SL, Annadoray L, Sian S, AckersJohnson MA, Foo RS: Targeting the highly abundant circular RNA circSlc8a1 in cardiomyocytes attenuates pressure overload induced hypertrophy. Cardiovasc Res. 2019.

59. Shan K, Liu C, Liu BH, Chen X, Dong R, Liu X, Zhang YY, Liu B, Zhang SJ, Wang JJ, et al. Circular noncoding RNA HIPK3 mediates retinal vascular dysfunction in diabetes mellitus. Circulation. 2017;136(17):1629-42.

60. Bachmayr-Heyda A, Reiner AT, Auer K, Sukhbaatar N, Aust S, BachleitnerHofmann T, Mesteri I, Grunt TW, Zeillinger R, Pils D. Correlation of circular RNA abundance with proliferation-exemplified with colorectal and ovarian cancer, idiopathic lung fibrosis, and normal human tissues. Sci Rep. 2015;5:8057.

61. Beltrami CA, Finato N, Rocco M, Feruglio GA, Puricelli C, Cigola E, Sonnenblick EH, Olivetti G, Anversa P. The cellular basis of dilated cardiomyopathy in humans. J Mol Cell Cardiol. 1995;27(1):291-305.

62. Khan MA, Reckman YJ, Aufiero S, van den Hoogenhof MM, van der Made I, Beqqali A, Koolbergen DR, Rasmussen TB, van der Velden J, Creemers EE, et al. RBM20 regulates circular RNA production from the Titin Gene. Circ Res. 2016;119(9):996-1003.

63. Bers DM. Cardiac excitation-contraction coupling. Nature. 2002;415(6868):198-205.

64. Herman DS, Lam L, Taylor MR, Wang L, Teekakirikul P, Christodoulou D, Conner L, DePalma SR, McDonough B, Sparks E, et al. Truncations of titin causing dilated cardiomyopathy. N Engl J Med. 2012;366(7):619-28.

65. Tang $Y$, Tian $X$, Wang R, Fill M, Chen SR. Abnormal termination of $\mathrm{Ca}^{2+}$ release is a common defect of RyR2 mutations associated with cardiomyopathies. Circ Res. 2012;110(7):968-77.

66. Salzman J, Chen RE, Olsen MN, Wang PL, Brown PO. Cell-type specific features of circular RNA expression. PLoS Genet. 2013;9(9):e1003777.

67. Hansen TB, Jensen TI, Clausen BH, Bramsen JB, Finsen B, Damgaard CK, Kjems J. Natural RNA circles function as efficient microRNA sponges. Nature. 2013;495(7441):384-8.

68. Salmena L, Poliseno L, Tay Y, Kats L, Pandolfi PP. A ceRNA hypothesis: the Rosetta Stone of a Hidden RNA language? Cell. 2011;146(3):353-8.

69. Rong D, Sun H, Li Z, Liu S, Dong C, Fu K, Tang W, Cao H. An emerging function of circRNA-miRNAs-mRNA axis in human diseases. Oncotarget. 2017:8(42):73271-81.

70. Chen JF, Mandel EM, Thomson JM, Wu Q, Callis TE, Hammond SM, Conlon FL, Wang DZ. The role of microRNA-1 and microRNA-133 in skeletal muscle proliferation and differentiation. Nat Genet. 2006;38(2):228-33.

\section{Publisher's Note}

Springer Nature remains neutral with regard to jurisdictional claims in published maps and institutional affiliations. 